\title{
Mechanisms driving alteration of the Landau state in the vicinity of a second-order phase transition
}

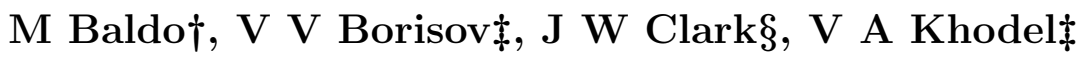 \\ and $M \mathrm{~V}$ Zverev $\ddagger$ \\ $\dagger$ Istituto Nazionale di Fisica Nucleare, Sezione di Catania, I-95123, Catania, Italy \\ $\ddagger$ Russian Research Center Kurchatov Institute, Moscow, 123182 Russia \\ $\S$ McDonnell Center for the Space Science and Department of Physics, Washington \\ University, St.Louis, MO 63130, USA
}

\begin{abstract}
The rearrangement of the Fermi surface of a homogeneous Fermi system upon approach to a second-order phase transition is studied at zero temperature. The analysis begins with an investigation of solutions of the equation $\epsilon(p)=\mu$, a condition that ordinarily has the Fermi momentum $p_{F}$ as a single root. The emergence of a bifurcation point in this equation is found to trigger a qualitative alteration of the Landau state, well before the collapse of the collective degree of freedom that is responsible for the second-order transition. The competition between mechanisms that drive rearrangement of the Landau quasiparticle distribution is explored, taking into account the feedback of the rearrangement on the spectrum of critical fluctuations. It is demonstrated that the transformation of the Landau state to a new ground state may be viewed as a first-order phase transition.
\end{abstract}

PACS numbers: $26.60 .+\mathrm{c}, 21.90 .+\mathrm{f}$

E-mail: zverev@mbslab.kiae.ru 


\section{Introduction}

In the Landau-Migdal theory of Fermi liquids [1, 2, the ground state of a homogeneous Fermi system is described in terms of a quasiparticle momentum distribution $n_{F}(p, T)$ that coincides with the momentum distribution of the ideal Fermi gas. This theory has been remarkably successful in advancing our qualitative and quantitative understanding of a broad spectrum of Fermi systems, including bulk liquid ${ }^{3} \mathrm{He}$, conventional superconductors, and nucleonic subsystems in neutron stars. However, the theory is known to fail in the strongly correlated electron systems present in high- $T_{c}$ compounds. Certain experimental results obtained very recently [3, 4, 5] may prove decisive to an understanding of this failure. The systems involved are a dilute two-dimensional (2D) electron gas and 2D liquid ${ }^{3} \mathrm{He}$. The experiments show how, under variation of the density, these systems progress from conditions of moderate correlation to the regime of very strong correlation. A striking feature is that both systems appear to experience a divergence of the effective mass $M^{*}$ as the density approaches a critical value $\rho_{\infty}$ associated with some kind of phase transition, which is presumably of second order [5].

We base our analysis on a necessary condition for the stability of the Landau state, namely that the change $\delta E_{0}$ of the ground state energy $E_{0}$ remain positive for any admissible variation from the quasiparticle distribution $n_{F}(p)=\theta\left(p_{F}-p\right)$, while keeping the particle number unchanged. Explicitly, this condition reads

$$
\delta E_{0}=\int \xi\left(p ; n_{F}\right) \delta n_{F}(\mathbf{p}) d \tau>0,
$$

for any variation $\delta n_{F}(\mathbf{p})$ satisfying

$$
\int \delta n_{F}(\mathbf{p}) d \tau=0
$$

In these equations, $d \tau$ is the volume element in momentum space, while $\xi(p)=\epsilon(p)-\mu$ is the single-particle (sp) spectrum, measured from the chemical potential $\mu$ and evaluated with the distribution $n_{F}(p)$.

The condition (11) holds provided the equation

$$
\xi(p)=0
$$

has the single root $p=p_{F}$. Otherwise, it is violated, the Landau state loses its stability, and the ground state must take another form, implying a rearrangement of singleparticle degrees of freedom. In weakly correlated Fermi systems, $\xi(p)$ is a monotonic function of $p$, so that equation (3) has no extra roots. However, as correlations build up, the character of the curve $\xi(p)$ may change. Indeed, it becomes non-monotonic in the vicinity of an impending second-order phase transition, when critical fluctuations of wave number $q_{c}>0$ produce a diverging susceptibility and hence a collapse of the corresponding collective degree of freedom.

Let the second-order phase transition occur at a critical density $\rho_{c}$. As we shall see, there is another critical density $\rho_{b}$ at which a bifurcation arises in equation (3), resulting in the emergence of a two additional roots $p_{1}, p_{2}$ (see figure 1). The distance between these extra roots increases linearly from zero in proportion to $\left|\rho-\rho_{b}\right|$. It should be 


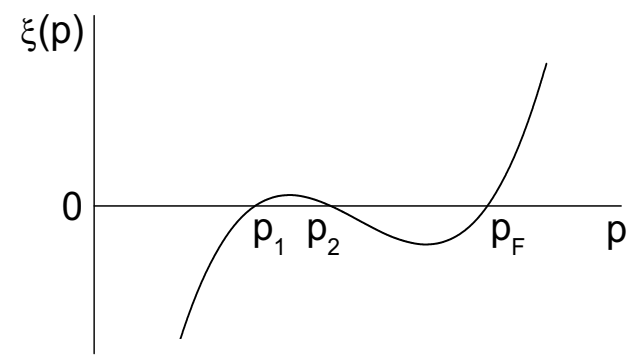

Figure 1. Illustration of the emergence of additional roots $p_{1}, p_{2}$ of equation (3).

emphasized that the stability condition (11) is never violated when applied to variations of the quasiparticle distribution $n(p)$ for momenta lying beyond the interval $\left[p_{1}, p_{2}\right]$. Hence, at $\left|\rho-\rho_{b}\right| \ll \rho$ the rearrangement process is confined to a constricted domain in momentum space. Accordingly, a rearrangement that entails a major alteration of the ground state in configuration space, involving all of the occupied sp states and therefore disfavored energetically and therefore irrelevant to the present study. In particular, Mott-Hubbard localization is ruled out.

For this reason our attention will be focused on two plausible scenarios for the rearrangement of the momentum distribution $n_{F}(p)$. In the first scenario, modification of the Landau state consists in the formation of empty spaces in momentum space that have been named Lifshitz bubbles (LB). In the LB phase, the quasiparticle occupation numbers have the usual values 0 and 1 , but the Fermi surface becomes multi-connected. In fact, this and related phenomena were studied in model problems more than 20 years ago [6, 7]. In the limit $\left|\rho-\rho_{b}\right| \ll \rho$, the LB mechanism has no rivals, provided the interval $\left[p_{1}, p_{2}\right]$ is not located in the immediate vicinity of the Fermi momentum $p_{F}$. Otherwise, there exists a novel competitor called fermion condensation [8, 9, 10, 11, 12, which is the second scenario to be examined here. Fermion condensation is a rearrangement of the Landau state leading from the Fermi step $n_{F}(p)$ to a continuous quasiparticle momentum distribution $n(p)$ having no Migdal jump at $p_{F}$. In the region $\mathrm{C}$ adjacent to the original Fermi surface where $n(p)$ departs from $n_{F}(p)$ by dropping smoothly from 1 to 0 , the sp spectrum turns out to be completely flat, with $\epsilon(p)=\mu$. This behavior gives rise to a singular, $\delta$-function term in the density of states $\rho(\varepsilon)$. Considered as a phase transition, fermion condensation does not break any symmetry, and has much in common with the classical gas-liquid phase transition [11. However, the presence of the singularity in $\rho(\varepsilon)$ enhances the feedback of the rearrangement process on the spectrum of the relevant critical fluctuations, which, in its turn, affects the competition between the two mechanisms proposed for rearrangement of the Landau state.

After investigating the nature of the instability of the Landau state, we shall illuminate the competition between LB and FC rearrangement scenarios by considering a simple model, in which the softening effect is assumed to depend linearly on the phase volume of region $\mathrm{C}$ occupied by the fermion condensate. It will be found that 
formation of the FC state exerts the greater impact on the collective degree of freedom. This being the case, we demonstrate that (i) the FC phase wins the contest with the LB reconfiguration, and (ii) the corresponding transformation of the Landau state is a first-order phase transition.

\section{Instability of the Landau state}

To gain detailed insight into the emergence of the bifurcation point in equation (3), we employ the Landau relation [1, 19]

$$
\frac{\partial \xi(p)}{\partial \mathbf{p}}=\frac{\mathbf{p}}{M}+\int f\left(\mathbf{p}, \mathbf{p}_{1}\right) \frac{\partial n\left(p_{1}\right)}{\partial \mathbf{p}_{1}} d \tau_{1},
$$

which connects the quasiparticle group velocity $d \xi / d p$ with the momentum distribution $n(p)$ in terms of the Landau scattering amplitude $f$.

First, we consider the case $q_{c} \sim p_{F}$, which applies to several phase transitions of fundamental interest. One of these is pion condensation, predicted to occur in (3D) neutron matter due to collapse of the collective spin-isospin mode with pion quantum numbers 13, 14, 15, 16. In this situation, the leading term in the amplitude $f$, being proportional to the singular term in the static spin-isospin susceptibility, has the form [13]

$$
f(q)=\frac{g}{\kappa^{2}(\rho)+\left(q^{2} / q_{c}^{2}-1\right)^{2}},
$$

where $g$ is a positive coupling constant and the stiffness coefficient $\kappa^{2}(\rho)$ vanishes at the critical density $\rho_{c}$. The same form of $f$ is expected to apply in two-dimensional liquid ${ }^{3} \mathrm{He}$, where spin fluctuations play an important role [17].

The sp spectrum $\xi(p)$ in the Landau state, with quasiparticle distribution $n_{F}(p)$, may be evaluated in closed form by means of equation (4). Substituting the expression (5) for the amplitude $f$ and performing the integration on the right-hand side, we obtain

$$
\begin{aligned}
\frac{d \xi\left(p, n_{F}\right)}{d p}= & \frac{p}{M}+\frac{g q_{c}^{4}}{16 \pi^{2} p^{2}}\left[\frac{1}{2} \log \frac{\left[\left(p-p_{F}\right)^{2}-q_{c}^{2}\right]^{2}+\kappa^{2} q_{c}^{4}}{\left[\left(p+p_{F}\right)^{2}-q_{c}^{2}\right]^{2}+\kappa^{2} q_{c}^{4}}\right. \\
& \left.+\frac{p^{2}+p_{F}^{2}-q_{c}^{2}}{\kappa q_{c}^{2}}\left(\arctan \frac{\left(p_{F}+p\right)^{2}-q_{c}^{2}}{\kappa q_{c}^{2}}-\arctan \frac{\left(p_{F}-p\right)^{2}-q_{c}^{2}}{\kappa q_{c}^{2}}\right)\right] .
\end{aligned}
$$

Further integration yields the formula

$$
\xi(p)=\xi^{0}(p)+\frac{g q_{c}^{2} p_{F}}{8 \pi^{2} \kappa} w(p),
$$

the dimensionless function $w(p)$ being given by

$$
\begin{aligned}
w(p) & =\frac{p_{F}^{2}-q_{c}^{2}-p^{2}}{2 p p_{F}}\left[\arctan \frac{\left(p_{F}+p\right)^{2}-q_{c}^{2}}{\kappa q_{c}^{2}}-\arctan \frac{\left(p_{F}-p\right)^{2}-q_{c}^{2}}{\kappa q_{c}^{2}}\right] \\
& -\frac{\kappa q_{c}^{2}}{4 p p_{F}} \ln \frac{\left[\left(p_{F}+p\right)^{2}-q_{c}^{2}\right]^{2}+\kappa^{2} q_{c}^{4}}{\left[\left(p_{F}-p\right)^{2}-q_{c}^{2}\right]^{2}+\kappa^{2} q_{c}^{4}} \\
& +\frac{\kappa q_{c}^{2}}{4 \sigma_{+} p_{F}}\left[\ln \left|\frac{\left(p_{F}+p\right)^{2}-2 \sigma_{+}\left(p_{F}+p\right)+\sigma_{0}^{2}}{\left(p_{F}+p\right)^{2}+2 \sigma_{+}\left(p_{F}+p\right)+\sigma_{0}^{2}}\right|+\ln \left|\frac{\left(p_{F}-p\right)^{2}-2 \sigma_{+}\left(p_{F}-p\right)+\sigma_{0}^{2}}{\left(p_{F}-p\right)^{2}+2 \sigma_{+}\left(p_{F}-p\right)+\sigma_{0}^{2}}\right|\right]
\end{aligned}
$$




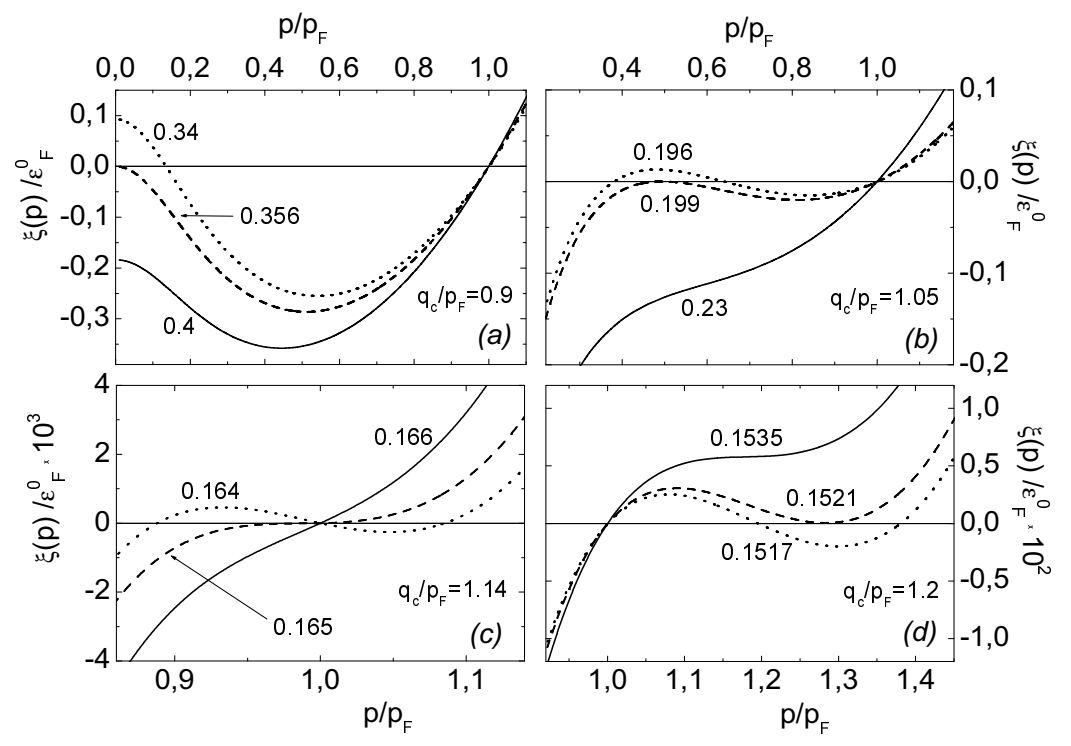

Figure 2. Neutron quasiparticle spectra $\xi(p)$ (in units of $\varepsilon_{F}^{0}$ ) evaluated for $q_{c}=0.9 p_{F}$ (panel (a)), $q_{c}=1.05 p_{F}$ (panel (b)), $q_{c}=1.14 p_{F}$ (panel (c)), and $q_{c}=1.2 p_{F}$ (panel (d)). Corresponding values of the parameter $\kappa$ are indicated near the curves.

$$
\begin{aligned}
+\frac{\kappa q_{c}^{2}}{2 \sigma_{-} p_{F}}\left[\arctan \frac{p_{F}+p+\sigma_{+}}{\sigma_{-}}+\arctan \frac{p_{F}-p+\sigma_{+}}{\sigma_{-}}\right. & \\
& \left.\left.+\arctan \frac{p_{F}+p-\sigma_{+}}{\sigma_{-}}+\arctan \frac{p_{F}-p-\sigma_{+}}{\sigma_{-}}\right)\right]
\end{aligned}
$$

where

$$
\sigma_{ \pm}=q_{c}\left(\frac{\sqrt{1+\kappa^{2}} \pm 1}{2}\right)^{1 / 2}, \quad \sigma_{0}=q_{c}\left(1+\kappa^{2}\right)^{1 / 4}
$$

and $\xi^{0}(p)=p^{2} / 2 M-\mu$.

Results of numerical calculations for neutron matter are shown in figures 2 and 3. For simplicity, we take the coupling constant in the amplitude (5) to be $g=1 / 2 m_{\pi}^{2}$, corresponding to bare $\pi N N$ vertices. The spectrum $\xi(p)$, evaluated with the critical momentum $q_{c}=0.9 p_{F}$ and for four values of the parameter $\kappa$, is displayed in panel (a) of figure 2

A new root $p_{b} \sim 0$ of equation (13) is seen to appear at $\kappa_{b} \simeq 0.356$, signaling that the Fermi step has become unstable. It is worth noting that at the customary values [13] of the critical momentum, $q_{c} / p_{F} \sim 0.7-1.0$, the bifurcation point lies exactly at the origin in $p$. However, as $q_{c}$ increases to greater values, it rapidly moves toward the Fermi momentum and leaves the Fermi sphere at $q_{c} \sim 1.14 p_{F}$. This evolution is illustrated by panels (b)-(d) of figure 2, where the spectra $\xi(p)$ calculated for $q_{c}=1.05 p_{F}, 1.14 p_{F}$, and $1.2 p_{F}$ are drawn. Figure 3 depicts the dependence $p_{b}\left(q_{c}\right)$ in the large interval $0<q_{c}<2 p_{F}$ (upper panel), together with the dependence of the critical parameter $\kappa_{b}$ on the wave number $q_{c}$ (lower panel). Remarkably, the largest values of $\kappa_{b}$ are achieved 

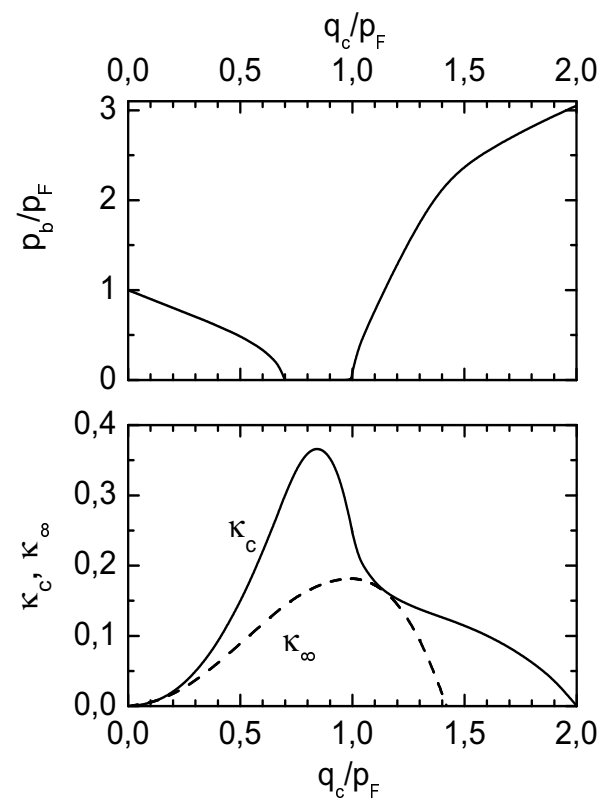

Figure 3. Upper panel: Position of the bifurcation point $p_{b}$ in units of the Fermi momentum, versus the critical wave number $q_{c}$ (also in units of $p_{F}$ ). Lower panel: critical parameters $\kappa_{b}$ and $\kappa_{\infty}$ as functions of $q_{c} / p_{F}$.

just in the preferred range $q_{c} / p_{F} \sim 0.7-1.0$. The value $\kappa_{\infty}$ of $\kappa$ at which the border of the instability region $\left[p_{1}, p_{2}\right]$ reaches the Fermi momentum $p_{F}$, is plotted in the lower panel of figure 3. The resulting curve lies below the curve of $\kappa_{b}\left(q_{c}\right)$ everywhere except for the point of contact at $q_{c} \simeq 1.14 p_{F}$.

The above results refer to the $3 \mathrm{D}$ problem. In the $2 \mathrm{D}$ case, analytical evaluation of the spectrum $\xi(p)$ is rather cumbersome, but its numerical computation is easily accomplished. We have calculated $\xi(p)$ for $2 \mathrm{D}$ liquid ${ }^{3} \mathrm{He}$ under the assumption that the dominant term in the quasiparticle amplitude is by governed by the static spinspin susceptibility. Results are shown in figure 4. While the spectrum of 2D liquid ${ }^{3} \mathrm{He}$ is found to differ quantitatively from that of 3D neutron matter, the shapes are qualitatively similar, as is the evolution with increasing $q_{c}$.

We infer from these two sets of results that in the case $q_{c} \lesssim p_{F}$, the Landau state becomes unstable prior to the second-order phase transition itself. As will be seen, this is a generic feature. On the other hand, particulars of the alteration of the Landau state will depend on the parameters that specify the amplitude $f$. To illustrate the general situation, we focus on a phase transition associated with the spontaneous generation of density waves in dense neutron matter or the dilute electron gas, in both of which the critical wave number $q_{c}$ is close to $2 p_{F}$. In this case, the scattering amplitude $f$ has the same form as (5), but the sign of $g$ is negative [11].

The spectrum $\xi(p)$ of the 3D electron gas, calculated for a critical momentum $q_{c}=1.95 p_{F}$ at three values of the parameter $\kappa$, is drawn in panel (a) of figure 5 . The solid line shows the spectrum at $\kappa \simeq 0.5015$, for which equation (3) has a single root 

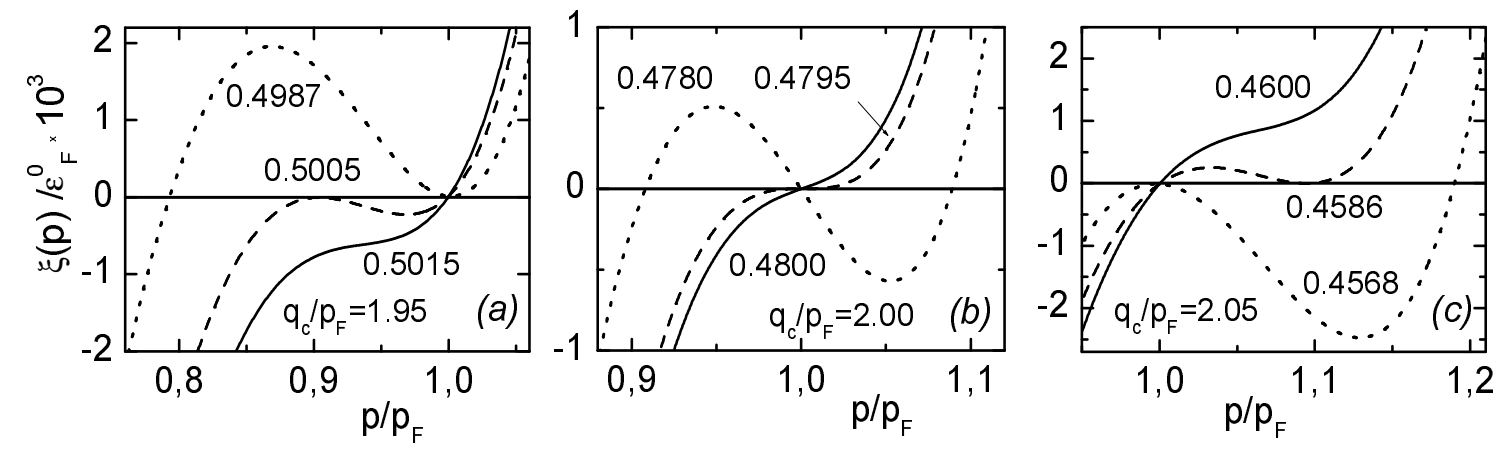

Figure 5. Electron spectra $\xi(p)$ in $3 \mathrm{D}$ (measured in units of $\varepsilon_{F}^{0}$ ), as calculated for $q_{c}=1.95 p_{F}$ (panel (a)), $q_{c}=2.00 p_{F}$ (panel (b)), and $q_{c}=2.05 p_{F}$ (panel (c)). The corresponding values of the parameter $\kappa$ are indicated near the curves. In each panel, the solid line traces the spectrum before the instability point is attained $\left(\kappa>\kappa_{b}\right)$, and the dotted line shows that at $\kappa<\kappa_{b}$. In the panels (a) and (c), the dotted line indicates the spectrum for $\kappa=\kappa_{\infty}$, at which the instability region reaches the Fermi surface.

at the Fermi momentum $p_{F}$. The long-dashed line depicts $\xi(p)$ at $\kappa=\kappa_{b} \simeq 0.5005$. As seen, the bifurcation point $p_{b}$ appears close to the Fermi momentum $p_{F}$. Also shown is the case when the bifurcation point reaches $p_{F}$ : the short-dashed line traces the sp spectrum at $\kappa_{\infty} \simeq 0.4987$, where the effective mass becomes infinite. This result was first obtained in reference [12]. The relevant plots for $q_{c}=2.0 p_{F}$ are displayed in panel (b). The solid line shows the spectrum at $\kappa \simeq 0.4800>\kappa_{b}$. For this choice of $q_{c}$, the bifurcation point $p_{b}$ appears exactly at the Fermi surface when $\kappa_{b} \simeq 0.4794$, as indicated by the long-dashed line. Since the effective mass goes to infinity, $\kappa_{\infty}$ and $\kappa_{b}$ coincide. The short-dashed line corresponds to a case beyond the critical point, with $\kappa \simeq 0.4780$. In all three cases, in the spectrum has a cubic-like shape as a function of $p-p_{F}$ in the vicinity of the Fermi momentum. The spectra for $q_{c}=2.05 p_{F}$ are shown in panel (c). 

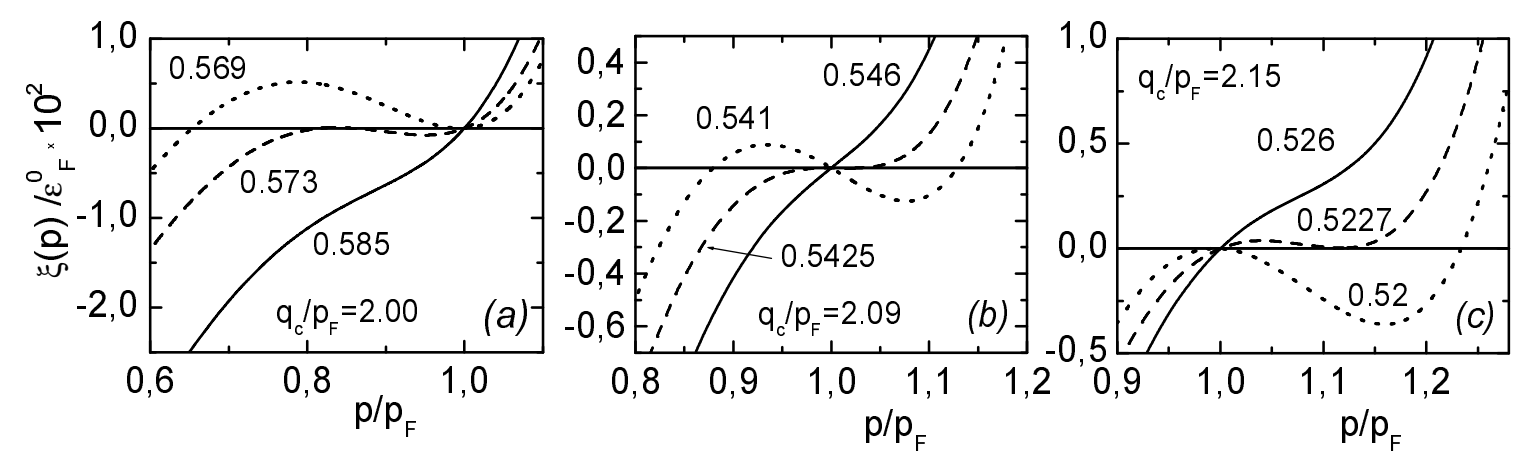

Figure 7. The same as in figure 5 but for the 2D case.

The solid line corresponds to $\kappa \simeq 0.4600$; the long-dashed line, to $\kappa_{b} \simeq 0.4586$; and the dotted line, to $\kappa_{\infty} \simeq 0.4568$. The bifurcation point is outside the Fermi sphere, with $p_{b} \sim 1.1 p_{F}$. The effective mass goes to infinity at $\kappa_{\infty}<\kappa_{b}$, as in the case of $q_{c}=1.95 p_{F}$. The behaviors of the parameters $p_{b}, \kappa_{b}$, and $\kappa_{\infty}$ as functions of the critical momentum $q_{c}$ are exhibited in figure [6. Analogous results are obtained in a study of ferromagnetic fluctuations, when $q_{c}=0$.

Calculation of the quasiparticle spectrum for $2 \mathrm{D}$ electron gas, with results collected in figure 7. confirms the implicit judgement that the 2D problem does not differ qualitatively from the 3D situation. 

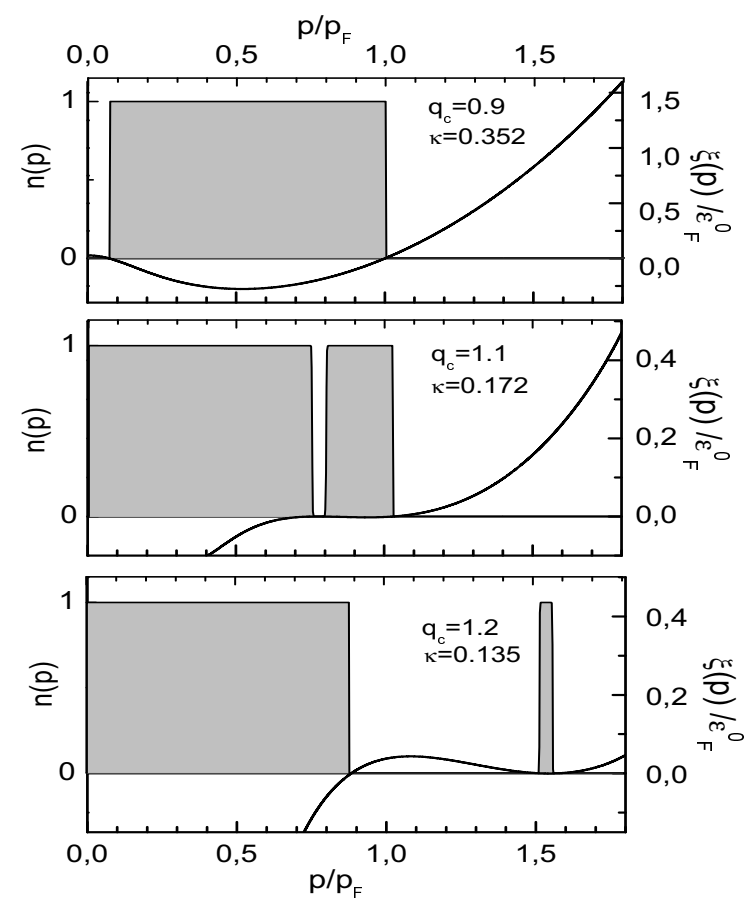

Figure 8. Neutron spectra $\xi(p)$ (in units of $\varepsilon_{F}^{0}$ ), together with the corresponding momentum distributions $n(p)$, calculated for $q_{c}=0.9 p_{F}$ and $\kappa=0.352$ (panel (a)), for $q_{c}=1.05 p_{F}$ and $\kappa=0.172\left(\right.$ panel (b)), and for $q_{c}=1.2 p_{F}$ and $\kappa=0.135$ (panel (c)).

\section{Competition between different rearrangement scenarios}

We now turn the discussion to the proposed scenarios for alteration of the Landau state beyond the limit of its stability, assuming that the difference $\left|\rho-\rho_{b}\right|$ is much smaller than $\rho$.

\subsection{Ignoring the feedback effects of rearrangement: Lifshitz-bubble formation}

In the pion-condensation example where $q_{c} \lesssim p_{F}$, we have seen that new roots of equation (31) arise quite far from the Fermi momentum $p_{F}$. As was shown in reference [18, the basic rearrangement mechanism transforming the Landau state in this case involves the formation of some number of the Lifshitz bubbles. The quasiparticle occupation numbers $n(p)$ remain integral at 0 or 1 , but the Fermi surface becomes multi-connected [6, 20].

Figure 8 presents some results from calculations designed to illustrate the characteristics of bubble formation. The sp spectra are evaluated by solving the closed equation for $\xi(p, T)$ that is obtained upon substitution of the Fermi-Dirac distribution $n(p, T)=[1+\exp (\xi(p, T) / T)]^{-1}$ into the r.h.s. of equation (4). Tuning of the chemical potential $\mu$ is governed by the normalization condition. A very small temperature $\left(T=10^{-5} \varepsilon_{F}^{0}\right)$ is used to imitate the zero-temperature case. The three panels in figure 8 show the neutron sp spectra calculated at three values of the critical momentum of spin- 


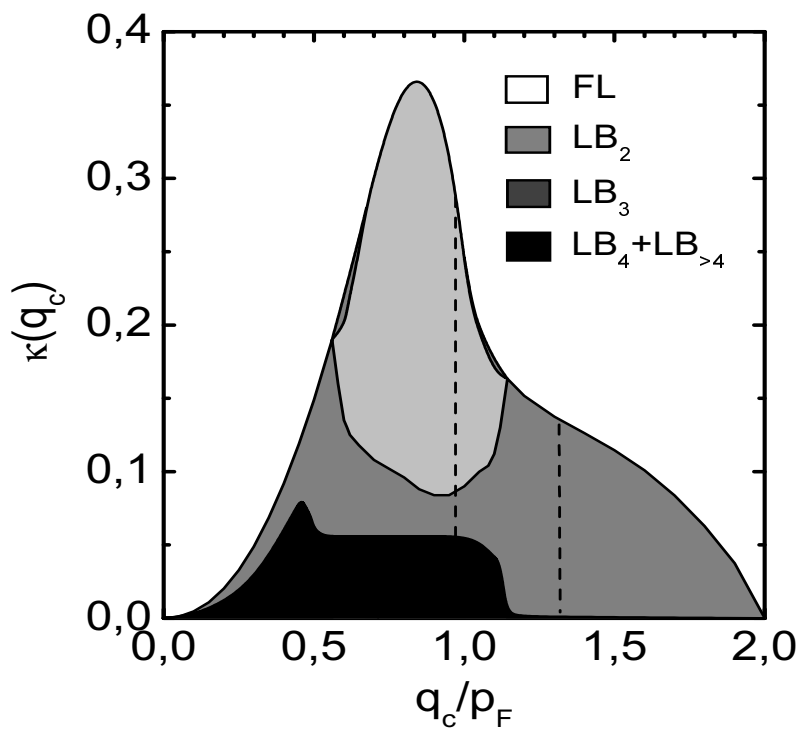

Figure 9. The phase diagram of neutron matter in $\left(q_{c}, \kappa\right)$ variables. The Landau phase (FL) occupies the white region of the plane. The LB phases are denoted by $\mathrm{LB}_{i}$, the index $i$ indicating the number of sheets (or branches) of the Fermi surface. Dashed lines show the borders of the territory occupied by the FC provided the feedback is taken into account.

isospin fluctuations, $q_{c}=0.9 p_{F}$ (panel (a)), $q_{c}=1.1 p_{F}$ (panel (b)), and $q_{c}=1.2 p_{F}$ (panel (c)). For all three parameter choices, the density $\rho$ is slightly above the critical value, and $n(p)$ exhibits a single LB, the position of which strongly depends on $q_{c}$. The bubble is located at the origin for $q_{c}=0.9 p_{F}$, at $p \sim 0.7 p_{F}$ for $q_{c}=1.1 p_{F}$, and mostly outside the original Fermi sphere for $q_{c}=1.2 p_{F}$. The bubble is small in cases (a) and (b), and the net disturbance relative to the original filled Fermi sea is small in all three cases.

As the density increases, the LB moves and multiplies. This behavior is demonstrated in figure 9. which shows the phase diagram of neutron matter in the $\left(q_{c}, \kappa\right)$ plane. The Landau state with $n(p)=n_{F}(p)$ occupies the white region of the diagram (labeled FL in the figure). The LB phases populate the shaded part of the plane, which is separated from the FL region by the curve $\kappa_{b}\left(q_{c}\right)$ (see figure 3). We shall not delve deeply into the "zoology" of the LB world, instead classifying the LB phases simply by the number $i$ of sheets of the Fermi surface.

Formation of Lifshitz bubbles is by no means the only kind of rearrangement the Fermi surface can experience as a result of the violation of stability condition (11). If the bifurcation point in equation (3) is situated close to the Fermi momentum $p_{F}$, then a new rearrangement scenario, fermion condensation [8, 9, 10, 11, comes into play. Its salient features are apparent from the basic equation

$$
\frac{\delta E}{\delta n(p)}=\mu, \quad p \in \mathrm{C} .
$$

This equation determines a new quasiparticle distribution $n_{0}(p)$ that differs from the 
Fermi distribution $n_{F}(p)$ within the region $\mathrm{C}$, but coincides with it outside. In contrast to the Lifshitz-bubble phases, the rearranged distribution $n_{0}(p \in \mathrm{C})$ appears to be a continuous function of $p$, with values lying between 0 and 1. Since its l.h.s. is nothing but the quasiparticle energy $\epsilon(p)$, the condition (10) implies the presence of a completely flat portion of the spectrum $\xi(p)$. This plateau in $\xi(p)$ identifies the fermion condensate (FC), i.e., the subsystem of quasiparticles with energy pinned to the chemical potential. As consequence of this behavior, the density of states $\rho(\varepsilon)$ acquires an infinite term at $\varepsilon=0$, as in a Bose liquid. It must be kept in mind, however, that the fermion condensation is in actuality an intermediate stage, since its inherent degeneracy must somehow be lifted. The analysis of this process is beyond the scope of the present article; a detailed treatment may be found in reference [11].

Equation (10) can be rewritten in explicit form by employing the well-known Landau formula

$$
\delta E=\delta^{(1)} E+\delta^{(2)} E
$$

for the variation of the ground state energy $E$ under variation $\delta n_{F}(p)=n(p)-n_{F}(p)$ of the Landau quasiparticle momentum distribution $n_{F}(p)$. Here

$$
\begin{aligned}
\delta^{(1)} E & =\int \xi\left(p ; n_{F}\right) \delta n_{F}(p) d \tau, \\
\delta^{(2)} E & =\frac{1}{2} \iint f\left(\mathbf{p}, \mathbf{p}_{1} ; n_{F}\right) \delta n_{F}(p) \delta n_{F}\left(p_{1}\right) d \tau d \tau_{1},
\end{aligned}
$$

where $f$ is the Landau amplitude entering equation (4). Insertion of this formula into condition (10) leads to the following equation for determining the new momentum distribution $n_{0}(p)$,

$$
\xi\left(p ; n_{F}\right)+\int f\left(\mathbf{p}, \mathbf{p}_{1} ; n_{F}\right)\left[n_{0}\left(p_{1}\right)-n_{F}\left(p_{1}\right)\right] d \tau_{1}=0 .
$$

Solutions of this equation can be assigned an order parameter $\eta$, taken as the ratio of the FC density to the total density $\rho$. Nontrivial solutions can arise beyond the point where the effective mass $M^{*}$ changes its sign. However, as we know from figure 7 Lifshitz bubbles already exist at this point. Thus, in the model adopted, LB states make their appearance prior to the formation of a fermion condensate.

To elucidate the situation, we may exploit the fact that in the region adjacent to the Fermi momentum $p_{F}$, the group velocity $d \xi / d p$ has essentially a parabolic shape. Defining a new variable $y=\left(p-p_{F}\right) / p_{F}$, we can write

$$
\begin{aligned}
& \frac{d \xi(y)}{d y} \simeq \frac{p_{F}^{2}}{M} A\left(3\left(y-y_{m}\right)^{2}+b\right), \\
& \xi\left(y ; n_{F}\right)=\frac{p_{F}^{2}}{M} A\left(\left(y-y_{m}\right)^{3}+b y+y_{m}^{3}\right) \equiv \frac{p_{F}^{2}}{M} A y\left(y^{2}-3 y y_{m}+3 y_{m}^{2}+b\right) .
\end{aligned}
$$

The three parameters $y_{m}$,

$$
A=\left.\frac{M}{6 p_{F}^{2}} \frac{d^{3} \xi(y)}{d y^{3}}\right|_{p_{F}}>0, \quad \text { and } \quad b=\left.\frac{M}{p_{F}^{2} A} \frac{d \xi(y)}{d y}\right|_{y_{m}}<0
$$


specifying the spectrum $\xi(p)$ depend on the parameter $\kappa$ appearing in the model form (5) for the Landau amplitude $f$. We observe that the parameter $b$ must be negative in the vicinity of the Fermi surface. At the point $\kappa=\kappa_{\infty}$ where the effective mass diverges, i.e. $(d \xi / d y)_{F}=0$, the parameters $y_{m}$ and $b$ are connected by the relation

$$
3 y_{m}^{2}\left(\kappa_{\infty}\right)+b\left(\kappa_{\infty}\right)=0 .
$$

On the other hand, the equation $\xi(y)=0$, with $\xi(y)$ given by the formula (15), has the single root $y=0$ for those $\kappa$ values at which

$$
s_{L B}(\kappa)=\frac{3 y_{m}^{2}(\kappa)}{4}+b(\kappa)>0 .
$$

Otherwise, the function $\xi(y)$ acquires two additional zeroes

$$
y_{1,2}=\frac{3 y_{m}(\kappa)}{2} \pm\left[-\left(\frac{3 y_{m}^{2}(\kappa)}{4}+b(\kappa)\right)\right]^{1 / 2},
$$

rendering the Landau state unstable. Setting $\kappa=\kappa_{\infty}$ in equation (15) and appealing to relation (17), we infer that at the point where fermion condensation sets in, the equation $\xi(y)=0$ already has three roots, namely $y_{1,2}=0$ and $y_{3}=3 y_{m}\left(\kappa_{\infty}\right)$. This confirms that the Landau state is unstable at the point of fermion condensation.

Thus, we have demonstrated both numerically and analytically that in the oversimplified model under consideration, alteration of the Landau state due to formation of Lifshitz bubbles does indeed precede fermion condensation. This property was first documented in the numerical calculations of reference [20].

3.2. A simple model including feedback: the contest between fermion condensation and Lifshitz-bubble creation

To this point, no consideration has been given to the effect of feedback on the critical fluctuations as reflected in their basic parameter, the stiffness coefficient $\kappa^{2}$ entering the interaction function $f(q)$ of equation (5). We now address this issue. Our analysis shows that the impact of Lifshitz-bubble formation on the critical fluctuations is insignificant. On the other hand, the feedback effect may be crucial in the case of fermion condensation, because of the infinite value taken by the density of states $\rho(\varepsilon=0)$ at $T=0$.

To provide a basis for analysis, we evaluate the gain in energy due to the emergence of a small FC fraction, assuming a trial FC function for the variation $\delta n(y)=n_{\mathrm{tr}}(p)-n_{F}(p)$ having the simplest form,

$$
\delta_{\operatorname{tr}} n(y)=\frac{1}{2} \operatorname{sgn} y, \quad-\lambda<y<\lambda .
$$

Particle number is conserved as long as the parameter $\lambda$ is sufficiently small. With this trial function, we evaluate the first- and second-order variations, $\delta_{\mathrm{tr}}^{(1)} E$ and $\delta_{\mathrm{tr}}^{(2)} E$, in the Landau formula (12). 
After inserting the trial function $\delta_{\text {tr }} n(p)$ along with the sp spectrum (15) into equation (12), simple manipulations yield

$$
\delta_{\mathrm{tr}}^{(1)} E(\lambda)=\frac{p_{F}^{2}}{M} \frac{\lambda^{2}}{4}\left[A \lambda^{2}+2 v_{g}\right],
$$

and

$$
\delta_{\mathrm{tr}}^{(2)} E=\frac{p_{F}^{2}}{M} \frac{B \lambda^{4}}{4},
$$

where we have introduced the dimensionless group velocity $v_{g}=A\left(3 y_{m}^{2}+b\right)$. Collecting terms, we arrive at

$$
\delta_{\mathrm{tr}} E=\frac{p_{F}^{2}}{M} \frac{\lambda^{2}}{4}\left[(A+B) \lambda^{2}+2 v_{g}\right],
$$

with $v_{g}=s_{L B}+9 y_{m}^{2} / 4$ and $s_{L B}$ given by relation (18).

As we have seen, the LB phase wins the contest with the Landau state if $s_{L B}<0$. To uncover the conditions under which the FC state can prevail in the competition between the two phases, let us investigate the roots of the function $\delta_{\operatorname{tr}} E(\lambda)$ given by equation (23). Quite evidently, if $v_{g}>0$, or equivalently, if $s_{L B}>-9 y_{m}^{2} / 4$, this function has no roots, and hence $\delta_{\mathrm{tr}} E(\lambda)>0$. This result demonstrates that without accounting for feedback of the FC on the stiffness coefficient $\kappa^{2}$, and hence on $v_{g}$, the FC phase looses the contest.

To proceed further we make the simple assumption that $v_{g}$ falls off linearly with increase of the FC density. Thus we write $v_{g}(\kappa, \lambda)=v_{0}(\kappa)-\lambda v_{1}(\kappa)$, where $v_{1}(\kappa)>0$ is a slowly varying function of $\kappa$. It is straightforward to show that equation $\delta_{\text {tr }} E=0$ has two positive roots

$$
\lambda_{1,2}=\frac{v_{1} \pm \sqrt{v_{1}^{2}-2(A+B) v_{0}}}{A+B},
$$

between which $\delta_{\text {tr }} E(\lambda)<0$ holds provided $v_{1}^{2}>2(A+B) v_{0}$. Therefore for any $\lambda$ within the range $\lambda_{1}<\lambda<\lambda_{2}$, the variation $\delta_{\operatorname{tr}} E(\lambda)$ is negative. Since the true value of $\delta_{0} E$, calculated with the true function $n_{0}(p)$ from equation (13), should lie lower, we infer that fermion condensation is energetically preferred over the Landau state - at least in the case that $v_{1}^{2}>2(A+B) v_{0}$. This inequality is always satisfied close to the point of fermion condensation, where according to equation (17), $v_{0}$ vanishes. Since both of the roots $\lambda_{1,2}$ are positive, not zero, fermion condensation is predicted to be a weak first-order phase transition.

In deciding the competition between the FC and $\mathrm{LB}$ phases, it is instructive to focus on the case of small positive $s_{L B}(\kappa)$, for which LB formation is still forbidden. The input parameters may be chosen so as to locate the minimum of $d \xi / d p$ not far from the Fermi surface, which implies a sufficiently small value of $y_{m}$. But at the point where $s_{L B}=0$, we have $v_{0}=9 y_{m}^{2} / 4$. Hence, if $y_{m}$ is sufficiently small, both of the roots $\lambda_{1}$, $\lambda_{2}$ of equation (24) are real, and $\delta_{\operatorname{tr}} E(\lambda)$ is negative in the interval between them. We then conclude that for $s_{L B} \rightarrow 0^{+}$, fermion condensation is allowed, while Lifshitz-bubble formation is forbidden. 


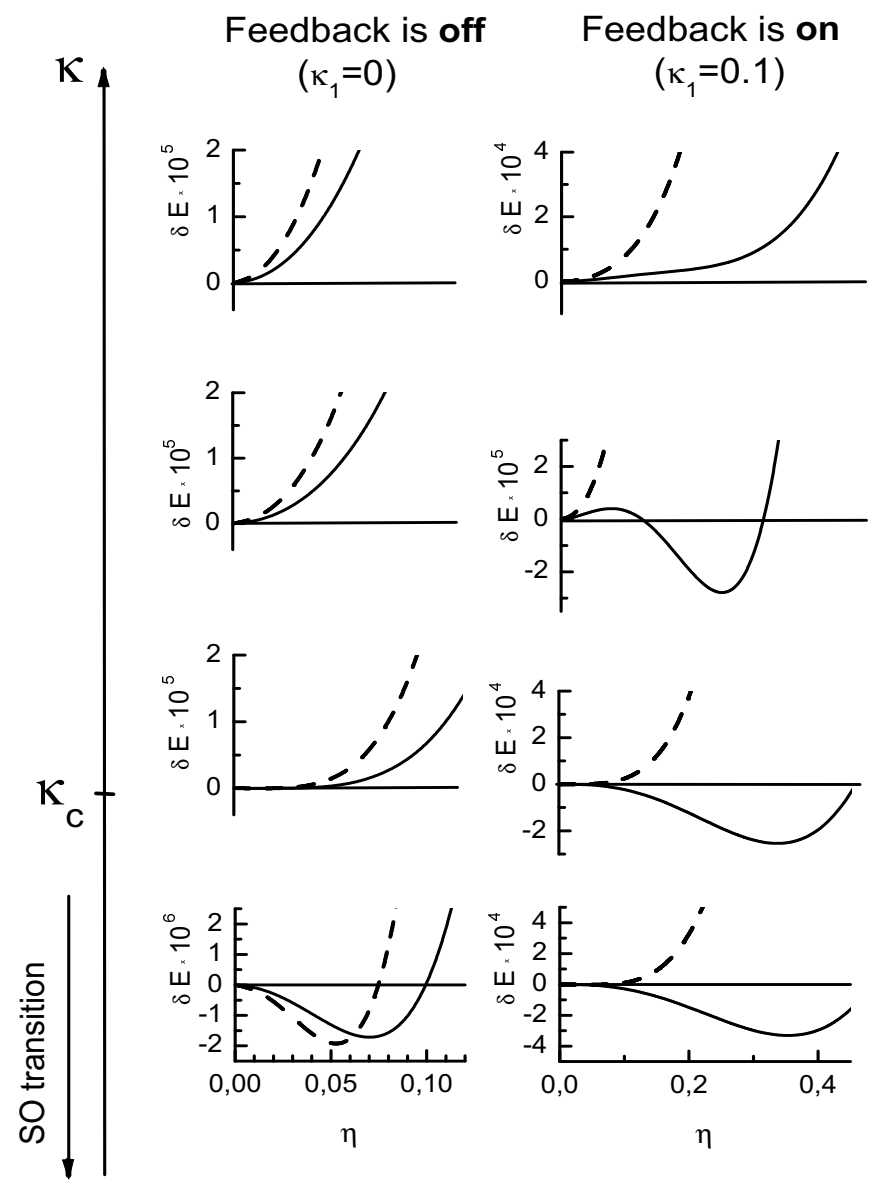

Figure 10. Energies (per particle) of the trial state $\delta_{\mathrm{tr}} E$ (solid line) and of the LB state $\delta_{\mathrm{LB}} E$ (dashed line), calculated for the 3D electron gas with $q_{c}=2 p_{F}$ and $\kappa_{0}=0.4820,0.4810,0.4794$, and 0.4790 (ordered from top to bottom). The energies, measured in units of the Fermi energy $\varepsilon_{F}^{0}$, are plotted versus the order parameter $\eta$. Left panels: feedback off $\left(\kappa_{1}=0\right)$. Right panels: feedback on $\left(\kappa_{1}=0.1\right)$.

\subsection{Numerical illustration}

The foregoing model analysis of the role of feedback in the competition between fermion condensation and Lifshitz-bubble formation can be illustrated by numerical calculation of the variation $\delta E$ of the ground-state energy corresponding to chosen variations $\delta n(p)$ of the quasiparticle distribution away from the Fermi distribution $n_{F}(p)$. (The same exercise will serve to demonstrate that the parameter $\alpha$ appearing in the secondorder energy variation (22) is indeed of order unity. We compare the energy variation corresponding to the $\mathrm{FC}$ trial variation $\delta_{\mathrm{tr}} n(p)$, with the energy variation $\delta_{\mathrm{LB}}$ associated with the LB phase. In figure 10, the energy shifts $\delta_{\mathrm{tr}} E$ and $\delta_{\mathrm{LB}} E$, evaluated for the 3D electron gas with $q_{c}=2 p_{F}$, are drawn as functions of the order parameter $\eta$, taken as the relative phase volume of the region in momentum space within which the quasiparticle distribution is rearranged. The left panels show the results obtained 
ignoring the suppression of the stiffness coefficient $\kappa^{2}$ due to formation of the FC. In this case, it is seen that both the FC trial state and the LB state give lower energy than the Landau state at $\kappa<\kappa_{b}$, but the LB state has the deeper minimum.

The feedback of the quasiparticle rearrangement on the charge fluctuations strongly alters the competitive balance between LB and trial FC states. For the trial FC state, the feedback effect is included in the same manner as detailed above. In particular, we assert a linear dependence on $\eta$ of the term $\kappa^{2}$ in the denominator of the amplitude (5), in the form $\kappa^{2}(\eta)=\kappa_{0}^{2}-\eta \kappa_{1}^{2}$. To be definite, we set $\kappa_{1}=0.1$. For the LB state, feedback is unimportant, since the density of states $\rho(\varepsilon)$ receives no dramatic enhancement in this rearrangement scenario. The right panels in the figure demonstrate the role of feedback in the competition between the three competing states. We observe that the plots of $\delta_{\text {tr }} E(\eta)$ differ markedly from their counterparts in the left panels, which represent the feedback-off situation. In accordance with the analysis of the previous section, a negative minimum of the curve $\delta_{\text {tr }} E$ first appears at a value of $\kappa$ below the critical value $\kappa_{c}$. Beyond $\kappa_{c}$ (right bottom panel), this minimum is lower than that of the LB curve by two orders of magnitude. Therefore, the state possessing the true $\mathrm{FC}$ - whose energy is necessarily below that of the trial FC state - clearly wins the contest with the LB phase, and the transition from the Landau state to the FC state is of first order.

Taking the feedback into account changes the phase diagram of neutron matter. The FC wins the contest with the LB states in the part of non-Landau area of the $\left(q_{c}, \kappa\right)$ phase diagram between two dashed lines in figure 9. We estimated these borders using the same parameter $\kappa_{1}=0.1$ as for the $3 \mathrm{D}$ electron gas.

\section{Conclusions}

Based on standard relations of the Landau theory of Fermi liquids, we have explored the properties of mechanisms that may force a rearrangement of the Fermi surface of a homogeneous system at zero temperature. It is found that in advance of a secondorder phase transition to a state with long-range order induced by the softening of the spectrum of critical fluctuations, there arise additional, nontrivial roots of the equation $\epsilon(p)=\mu$, signaling an instability of the Landau state. The consequent metamorphosis of the quasiparticle spectrum has been traced to a divergence, at the second-order transition point, of the leading term in the quasiparticle amplitude, which is proportional to the pertinent static susceptibility.

We have clarified the competitive status of two scenarios for alteration of the Landau state, Lifshitz-bubble formation and fermion condensation. In general, and in particular for the case of fermion condensation, it must be expected that the rearrangement of the quasiparticle momentum distribution will exert an influence on the implicated collective degree of freedom. This feedback effect has been taken into account through a simple model in which the stiffness coefficient depends linearly on the FC density. Without feedback, Lifshitz-bubble formation precedes fermion condensation. However, the introduction of feedback reverses this picture: under increase of density, the first- 
order phase transition to the state containing a fermion condensate takes place before bubble-formation becomes the favored state.

\subsection{Acknowledgments}

This research was supported in part by the National Science Foundation under Grant No. PHY-0140316 (JWC and VAK), by the McDonnell Center for the Space Sciences at Washington University (VAK), by Grant No. NS-1885.2003.2 from the Russian Ministry of Industry and Science (VVB, VAK, and MVZ), by Fellow Grants from Russian

Research Centre "Kurchatov Institute" (VVB and MVZ) and by a Student Grant from the "Dynasty" Foundation (VVB). Two of the authors (VVB and MVZ) thank INFN (Sezione di Catania) for hospitality during their stay in Catania.

\section{References}

[1] Landau L D 1956 Sov. Phys.-JETP 301058 Landau LD 1958 Sov. Phys.-JETP 3597

[2] Migdal A B 1967 Theory of Finite Fermi Systems and Applications to Atomic Nuclei (New York: Wiley)

[3] Casey A, Patel H, Nyeki J, Cowan B P and Saunders J 2003 Phys. Rev. Lett. 90115301

[4] Kravchenko S V and Sarachik M P 2004 Rep. Prog. Phys. 671

[5] O. Prus, Y. Yaish, M. Reznikov, U. Srivan and V. M. Pudalov 2003 Phys. Rev. B 67205407

[6] De Llano M and Vary J P (1979) Phys. Rev. C 191083

De Llano M, Plastino A and Zabolitsky J G (1979) Phys. Rev. C 202418

[7] Aguilera-Navarro V C, De Llano M, Clark J W and Plastino A (1982) Phys. Rev. C 25560

[8] Khodel V A and Shaginyan V R (1990) JETP Lett. 51553

[9] Volovik G E (1991) JETP Lett. 53222 Volovik G E (1994) JETP Lett. 59830

[10] Nozieres P (1992) J. Physique I 2449

[11] Khodel V A, Shaginyan V R and Khodel V V (1994) Phys. Rep. 2491

[12] Khodel V A, Shaginyan V R and Zverev M V (1997) JETP Lett. 65254

[13] Migdal A B (1978) Rev. Mod. Phys. 50107

[14] Migdal A B, Saperstein E E, Troitsky M A and Voskresensky D N (1998) Phys. Rep. 192179

[15] Akmal A, Pandharipande V R and Ravenhall D G (1998) Phys. Rev. C 581804

[16] Wiringa R B, Fiks V and Fabrocini A (1988) Phys. Rev. 381010

[17] Boronat J, Casulleras J, Grau V, Krotscheck E and Springer J (2003) Phys. Rev. Lett. 91085302

[18] Voskresensky D N, Khodel V A, Zverev M V and Clark J W (2000) Ap. J. Lett. 533127

[19] Abrikosov A A, Gor'kov L P, Dzyaloshinskii I Y 1990 Quantum Field Theoretical Methods in Statistical Physics (New York: Pergamon Press)

[20] Zverev M V and Baldo M 1998 JETP 871129 Zverev M V and Baldo M 1999 J. Phys.: Condens. Matter 112059 